\title{
NIGHTTIME SPEED NEGOTIATION ON RURAL ROAD S-SHAPED CURVES: DISCUSSION OF AN EXPERIMENTAL CASE-STUDY
}

\author{
Marco Pasetto and Andrea Manganaro \\ Department of Structural and Transportation Engineering \\ University of Padua \\ Padova (Italy) \\ Email: marco.pasetto@unipd.it; andrea.manganaro@dic.unipd.it
}

\begin{abstract}
Summary: Road users' perception of risk while driving is the focal point discriminating prudent from imprudent behavior. One of the main factors that can influence a driver's level of risk perception is 'lighting', given that the driving conditions on roads alter radically between daytime and nighttime hours. This paper describes the results of a study conducted along a section of rural road containing a sharp S-shaped curve linking two long rectilinear approach roads in the sequence 'tangent-curve-tangent'. The driving behavior of the road users was surveyed in both directions of travel by day and at night, demonstrating a notable discrepancy in driving style between the two conditions.
\end{abstract}

\section{INTRODUCTION}

The behavior of road users while driving derives from the interaction of a series of both endogenous and exogenous factors: on the one hand are the personal motivations and psychophysical state of the driver, on the other the influencing variables deriving from the road environment. Among the best studied influencing factors (Bella, 2006; Diete et al., 2005; Fink \& Krammes, 1995), the geometric characteristics of the route contribute significantly. The effects of road signs and road markings on drivers' behavior (Charlton, 2004, 2007) have also been widely investigated, especially in the phase of negotiating the speed of approach to horizontal curves. Inevitably, the driving behavior of road users is also strongly affected by perceptive and attentional factors closely connected to the geometric and functional characteristics of the roads. A lack of experience and the inability to manage complex situations (McDonald \& Ellis, 1975) are compounded by an underestimation of speed and actual curvature of the road (Johnston, 1982; Messer et al., 1981), distraction by external factors (use of a cell phone or radio) (Charlton, 2004) and the level of tiredness or visual capacity of the driver in the different conditions.

One of the factors with most influence on road safety is 'lighting', given that the behavior of road users varies markedly between daytime and nighttime conditions, with the fatal accident rates increasing from two to three times in the latter case (Blanco, 2002). By its very nature, nighttime driving is much less safe than daytime, especially when the illumination seen by the driver comes exclusively from the vehicle's headlamps, with the consequence that the only information useful for the driver's choice of conduct is derived from a very limited area of the road space (Olson, 1993), while background information is more or less absent (Fildes, 1993). Under these circumstances the perception of external risks, as well as interpretation of the road space, is extremely limited. The risk is further accentuated when the reduction of the users' risk perception is accompanied by inappropriate driving behavior, with traveling speeds unvaried 
with respect to the daytime, if not higher (Blanco, 2002; Olson, 1993), despite the limited visibility (Fildes, 1989).

\section{METHODOLOGICAL APPROACH}

With the aim of evaluating the effect of the factor 'lighting' on driving behavior, with other conditions being equal, a study context was identified with the following criteria: 1) axis geometry composed of a sequence of the type 'tangent-curve-tangent' without transition curves between successive geometric elements; 2) curvilinear section composed of two sharp curves ( $\mathrm{R}$ $<100 \mathrm{~m}$ ); 3) presence of geometric and functional defects that could affect the driving conduct of the users; 4) almost total absence of artificial lighting, at least along the approach tangents to the S-shaped stretch.

The layout was composed of two long rectilinear roads between which an S-shaped planimetric variant had recently been inserted, composed of two opposite horizontal curves linked by a short rectilinear stretch without interposing transition curves. Given that on the rectilinear sections (tangents) the roadway is composed of a single carriageway $5.50 \mathrm{~m}$ wide for both directions of travel and that the S-shaped planimetric variant has two $3.50 \mathrm{~m}$ traffic lanes, one in each direction, the geometric characteristics of the studied stretch are summarized in the table below.

Table 1. Geometric characteristics of the stretch of road.

\begin{tabular}{lcc}
\hline Road geometry & Length $(\mathbf{m})$ & Radius $(\mathbf{m})$ \\
\hline Tangent 1 & 166.3 & - \\
Curve 1 & 56.04 & 245.44 \\
Curve 2 & 83.90 & 96.49 \\
Rectilinear transition stretch & 26.20 & - \\
Curve 3 & 69.57 & 81.03 \\
Tangent 2 & 404.60 & - \\
\hline
\end{tabular}

The most interesting elements in terms of a behavioral evaluation are the presence of a new road intersection at the entrance to one of the two horizontal curves, which introduces a potential error in the perception of the layout approaching from the east, further aggravated by the position of the public road lighting that does not allow an efficient interpretation of the physiognomy of the route during the night.

With the aim of analyzing the users' driving behavior, 8 sections were identified for monitoring passing traffic, where speeds were monitored by video-camera in both directions of travel, during the daytime and at night. These sections corresponded to the above-described geometric elements and were numbered from 1 to 8 , traveling on the route from west to east. Section 1 is midway along the tangent of approach (MT1) to the first horizontal curve. Sections 2 and 6 (PC1 and PC3) are positioned at the entry points to the S-shaped bend. Sections 3, 4 and 5 are at the mid-points of the three horizontal curves (MC 1, MC2, MC3) and sections 7 and 8 are midway and at the eastern end of tangent 2 (MT2 and PT2).

The speed surveys were conducted through the acquisition and subsequent photogrammetric scansion of the footage of passing vehicles taken by video-cameras hidden at the roadside. The measurements were limited to isolated light vehicles only - with a reciprocal distance of no less 
than 4 seconds - in conditions of clear weather and dry road surface. The filming was done at the same times for all the sections during the daytime and at night.



Figure 1. Position of the speed monitoring stations

\section{RESULTS}

The speed surveys conducted in the daytime included a total of 4215 vehicles, with an average of approximately 250 per direction of travel on each section. Regarding the nighttime, the projection of the results is based on a total of 2885 vehicles, corresponding to an average of approximately 180 transits per direction on each of the sections.

The curves of cumulative distribution of the users' speeds were calculated for each section, from which the $85^{\circ}$ percentile value of the speed was taken. This is descriptive of the driving speed on the geometric element considered and is typically used in the literature to describe the driving behavior of road users.

\section{Direction A}

The speed profile for the direction of travel A shows a net daytime/nighttime discrepancy in driving conduct on the S-shaped stretch (from $\mathrm{MC} 1$ to $\mathrm{MC} 3$ ), passing from an average close to $60 \mathrm{~km} / \mathrm{h}$ in daylight conditions to values of between 50 and $55 \mathrm{~km} / \mathrm{h}$ at night.

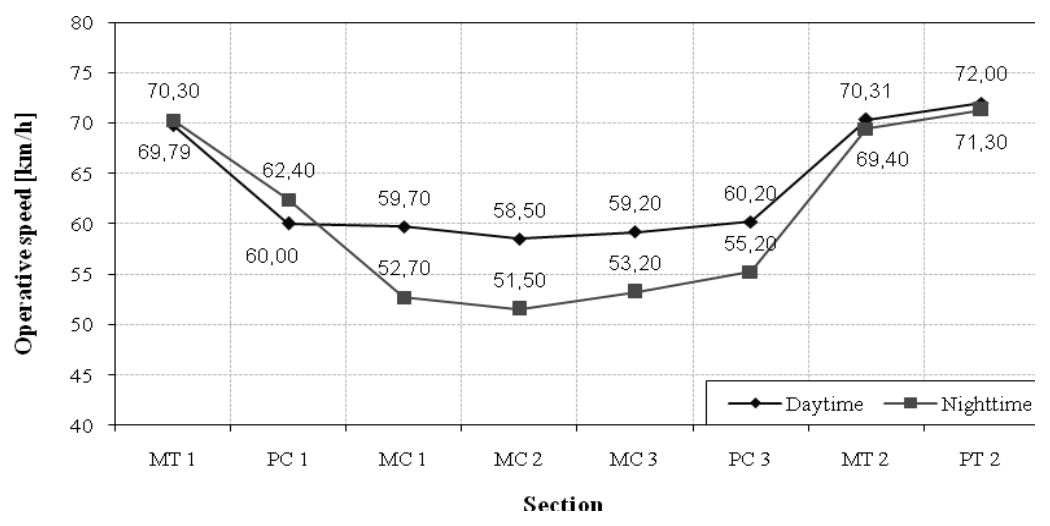

Figure 2. Profile of the driving speed in direction of travel A 
The table of average accelerations calculated between the sections demonstrates the adjustment of speed on the curve (PC1 - MC1) during the daytime, which corresponds to an acceptable deceleration in absolute value. The rapid acceleration as the users exit the last curve (PC3 MT2) during the night should be noted, which corresponds to a final speed at MT2 almost equal to the daytime one, probably considered as the most comfortable and suitable for the type of road, even at night.

Table 3. Summary of average accelerations between successive sections in direction of travel A

\begin{tabular}{cccccc}
\hline \multirow{2}{*}{$\begin{array}{c}\text { Section } \\
\text { No. }\end{array}$} & \multirow{2}{*}{ Description } & \multicolumn{4}{c}{ Acceleration/Deceleration [m/s2] } \\
\cline { 3 - 6 } & & Daytime & Nighttime & Difference & $\%$ \\
\hline $1-2$ & MT 1 - PC 1 & -0.37 & -0.30 & 0.07 & $-19.3 \%$ \\
$2-3$ & PC 1 - MC 1 & -0.01 & -0.16 & -0.16 & $3133.3 \%$ \\
$3-4$ & MC 1 - MC 2 & -0.09 & -0.09 & 0.00 & $0.0 \%$ \\
$4-5$ & MC 2 - MC 3 & 0.10 & 0.24 & 0.14 & $142.9 \%$ \\
$5-6$ & MC 3 - PC 3 & 0.18 & 0.37 & 0.18 & $100.0 \%$ \\
$6-7$ & PC 3 - MT 2 & 0.78 & 1.10 & 0.32 & $40.5 \%$ \\
$7-8$ & MT 2 - PT 2 & 0.05 & 0.05 & 0.01 & $12.4 \%$ \\
\hline
\end{tabular}

\section{Direction B}

In the opposite direction of travel, the users are faced with the visual difficulties on section MC3, because of the presence of the new intersection (see figure 1) and the position of the road lighting which - on that side - continues along the spur leaving the intersection instead of following the curve. The speeds measured during the night clearly show the visual impact produced by these defects (stretch PC3 - MC3) compared to those observed in conditions of natural daylight.

The figure below confirms that the users tend to want to maintain driving speeds on the straight stretch of the same order of magnitude as those in the daytime, as also demonstrated by the brusque acceleration when leaving the curve (PC1 - MT1), corresponding to an even higher final speed than that measured during the hours of daylight. The speeds on the double bend remain $15 \%$ lower on average than those measured during the day. Those observed on the two tangents are instead 3-5\% higher on average.

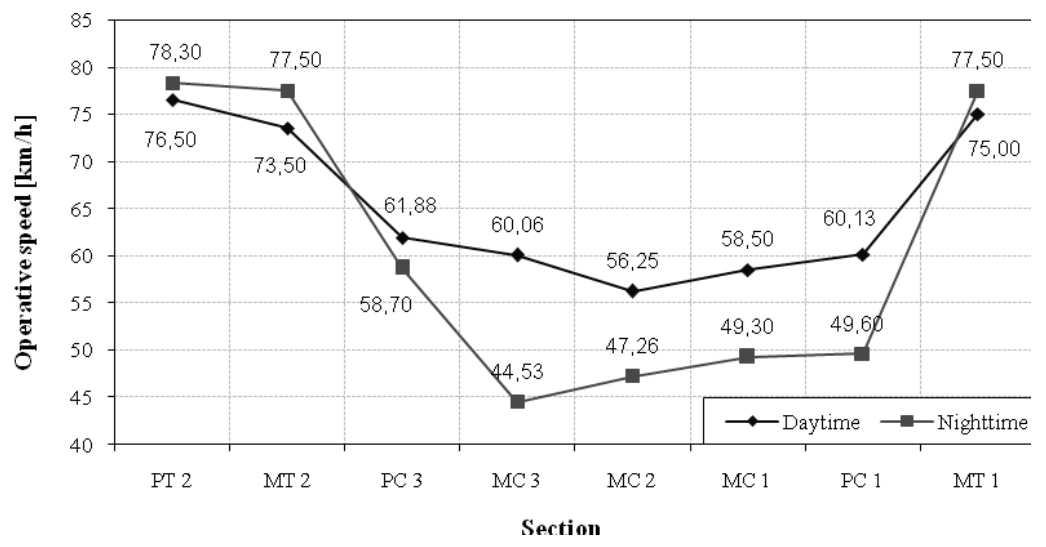

Figure 4. Speed profile in direction of travel B 
The table summarizing the accelerations shows that the users tend to underestimate the risk inherent in the double bend until they are entering it, at which point - faced with the visual ambiguity caused by the road geometry - they decelerate significantly $\left(-2.66 \mathrm{~m} / \mathrm{s}^{2}\right)$ to reduce their speed to one considered more appropriate to the belatedly perceived risk.

Table 5. Summary of average accelerations between successive sections in direction of travel B.

\begin{tabular}{cccccc}
\hline \multirow{2}{*}{$\begin{array}{c}\text { Section } \\
\text { No. }\end{array}$} & \multirow{2}{*}{ Description } & \multicolumn{4}{c}{ Acceleration/Deceleration [m/s2] } \\
\cline { 3 - 6 } & & Daytime & Nighttime & Difference & $\%$ \\
\hline $8-7$ & PT 2 - MT 2 & -0.09 & -0.02 & 0.07 & $-73.3 \%$ \\
7 - 6 & MT 2 - PC 3 & -0.93 & -1.51 & -0.58 & $61.8 \%$ \\
$6-5$ & PC 3 - MC 3 & -0.34 & -2.66 & -2.31 & $678.6 \%$ \\
$5-4$ & MC 3 - MC 2 & -0.53 & 0.38 & 0.92 & $-171.7 \%$ \\
$4-3$ & MC 2 - MC 1 & 0.17 & 0.15 & -0.02 & $-9.3 \%$ \\
$3-2$ & MC 1 - PC 1 & 0.03 & 0.01 & -0.02 & $-81.6 \%$ \\
$2-1$ & PC 1 - MT 1 & 0.59 & 1.10 & 0.51 & $87.6 \%$ \\
\hline
\end{tabular}

\section{CONCLUSIONS}

The capacity of drivers to correctly negotiate their speed when approaching curves is closely correlated to the number and seriousness of accidents on horizontal curves, especially when visibility is reduced. Visual sensibility during nighttime hours is much lower and statistically concentrated just on stimuli arriving from the roadway, preferably if deriving from the road markings and street lighting (Wonho Suh et al., 2006). It is obvious that if these stimuli are incorrect or difficult to interpret the risk of human error increases considerably, as well documented by the attitude at curve 3 along direction B, in regard to which: starting from a considerable rectilinear speed (PT2), even faster than that registered during the daytime on the same section, the user tends to maintain it even where there are road signs (MT2) warning of the double bend, with a negligible deceleration between the two sections $\left(-0.02 \mathrm{~m} / \mathrm{s}^{2}\right)$. Heading towards the curve (PC3), the user decelerates significantly $\left(-1.51 \mathrm{~m} / \mathrm{s}^{2}\right)$, reducing to speeds close to those of the daytime $(58.70 \mathrm{~km} / \mathrm{h})$, but as these are still considered excessive with respect to the perceived risk, there is a further marked deceleration $\left(-2.66 \mathrm{~m} / \mathrm{s}^{2}\right)$ on the horizontal curve (PC3 - MC3) with the resulting requirement for transverse adhesion. On the rest of the S the driver marginally adjusts his speed, increasing it until he exits curve 1 (PC1), where he accelerates vigorously to regain the speed considered more suitable and comfortable (77.50 $\mathrm{km} / \mathrm{h}$ ) according to the risks experienced and perceived along the route. During the daytime, driving speed along the tangents being equal, the user adopts more homogeneous behavior (although associated to higher speeds). In particular, $86 \%$ of the reduction in speed - and therefore the negotiation of the speed on the curve in relation to the perceived risk - occurs between sections MT2 and PC3 and only 14\% on the horizontal curve, as proof of a better interpretation of the information (although erroneous) from the road space. On the contrary, during the night only $57 \%$ of the deceleration is done along the transition stretch (MT2 - PC3), while the remaining $43 \%$ is dissipated on curve 3 , showing how the erroneous information coming from the road space (intersection and illumination) can alter the perception while driving, especially when the user has no other elements coming from the background useful for interpreting the risks. 
In the opposite direction (direction A), the differences between the daytime and nighttime scenarios in terms of risk perception and driving behavior are as follows: as in the previous case, the driving speed measured along the two tangents is of the same order of magnitude as the daytime one, if not actually higher (MT1); the driving speeds along the S are $10 \%$ lower on average than those in daytime (average $51-52 \mathrm{~km} / \mathrm{h}$ ); the perception of the curvature is delayed to the point that only $45 \%$ of the deceleration from the approach speed to that for negotiating the curve is done along the tangent and a good 55\% on the horizontal curve. During the daytime, on the contrary, against a higher speed on the curve (approx. $59 \mathrm{~km} / \mathrm{h}$ ), the deceleration is completed on the tangent, while the $\mathrm{S}$ is transited at practically constant speed.

In conclusion, these observations appear to confirm the users' preference to drive at night as they do in the daytime, if not at even higher speeds. The presence of narrow radius curves - which require greater attention and ability in the phase of negotiation of the driving speed - after long rectilinear tangents shows a marked discrepancy in driving behavior between the daytime and nighttime hours: while the daytime driving speeds on the straight are on average equivalent to those at night, the difference on the curve is clear, with daytime values approximately $10-15 \%$ higher than those at night. The greater risk is associated with the drivers' difficulty in interpreting the route geometry, as they do not tend to adjust their speed along the tangent (as they do by day) but also on the horizontal curve, demonstrating on the one hand an excessive underestimation of the risks associated with nighttime driving and on the other the extreme vulnerability of some geometric configurations in less than ideal conditions.

\section{REFERENCES}

Bella, F. (2006). Effects of driver perception of combined curves on speed and lateral placement, Proceedings of the $85^{\text {th }}$ Annual Meeting Transportation Research Board, January 22-26, 2006, Washington D.C.

Blanco, M. (2002). Relationship between driver characteristics, nighttime driving risk perception, and visual performance under adverse and clear weather conditions and different vision enhancement systems, Diss. submitted to the faculty of the Virginia Polytechnic Institute and State University.

Charlton, S.G. (2004). Perceptual and attentional effects on drivers speed selection at curves. Acc. An. and Prev., 36, 877-884.

Charlton, S.G. (2007). The role of attention in horizontal curves: A comparison of advance warning, delineation, and road marking treatments. Acc. An. and Prev., 39(5), 873-885.

Fildes, B.N. - Leening, A.C. - Corrigan, J.McM. (1989). Speed perception: drivers' judgements of safety and speed on rural straight and curved roads and for different following distances, Report no. CR60, Dept. of Transport, Federal Office of Road Safety, Victoria 3174, Australia.

Fildes, B.N. \& Lee, S.J. (1993). The speed review: road environment, behaviour, speed limits, enforcement and crashes, Report no. CR127 (FORS), Federal Office of Road Safety, Canberra, Australia.

Fink, K.L. \& Krammes, R.A. (1995). Tangent length and sight distance effects on accident rates at horizontal curves on rural two-lane roads, Transp. Res. Rec., 1500, 162-168. 
Johnston, I.R. (1982). Modifying driver behaviour on rural road curves: a review of recent research. Proc. of the 11th Australian Road Research Board (ARRB) Conference, Vol. 11(4), 115-124.

McDonald, L.B. \& Ellis, N.C. (1975). Driver workload for various turn radii and speeds, Transp. Res. Rec., , 530, 8-30.

Messer, C.J. - Mounce, J.M. - Brackett, R.Q. (1981). Highway geometric design consistency related to driver expectancy. Publication No. FHWA-RD-81-035. Federal Highway Administration, Washington. DC.

Olson, P. L. (1993). Vision and Perception. In B. Peacock, and W. Karwowski (Eds.), Automotive Ergonomics. 299-320, London: Taylor \& Francis.

Olson, P. L., \& Sivak, M. (1983). Comparison of headlamp visibility distance and stopping distance. Perceptual and Motor Skills, 57, 1177-1178.

Wonho Suh - Young-Jin Park, P. - Chang Ho Park - Kyung Soo Chon (2006). Relationship between speed, lateral placement, and drivers' eye movement at two-lane rural highways, Journal of Transportation Engineering, 132 (8), 649-653. 